\title{
Détection et mesure de la grêle par radar
}

\section{Detecting and measuring hail by radar}

\author{
A. Waldvogel \\ Ecole polytechnique fédérale \\ Département Physique de l'atmosphère, Zurich, Suisse
}

\section{Introduction}

Depuis le début de la radar-météorologie, la détection et la mesure de la grêle par radar ont toujours suscité un grand intérêt. La prévision des orages et les recherches scientifiques portant sur la lutte contre la grêle avaient un besoin urgent d'une telle technique.

Un des premiers critères permettant d'identifier des nuages porteurs de grêle, et cela dès la formation d'une cellule convective, avait été développé par des scientifiques soviétiques préoccupés par les dégats dûs à la grêle. Ce critère était fondé sur les dimensions géométriques des nuages. A l'origine, les mesures étaient plutôt effectuées au moyen d'instruments d'optique que par radar. Ce critère et sa validité seront examinés au chapitre 2.

Depuis 1960, les méthodes de détection et de mesure de la grêle ont été améliorées par l'emploi :

- de radars à une seule longueur d'onde de $10 \mathrm{~cm}$ (Geotis 1963);

- de radars à plusieurs longueurs d'onde "multiwavelength" (Atlas et Ludlam 1961);

- et depuis peu, par des radars à différentes directions de polarisation (Seliga et Bringi 1976 - Barge 1972).

Les problèmes pratiques se sont révélés beaucoup plus importants qu'ils n'avaient été jugés dès l'abord. En effet, en examinant les données enregistrées par le système radar à deux longueurs d'onde, Rinehart et Tuttle (1982) ont constaté que les mesures devaient être considérées comme douteuses en raison des imperfections techniques du système. Cette remarque est également valable, en partie, pour les travaux d'Eccles (1979) comparant les données du radar à celles des grêlimètres.

Ceci est très regrettable car, à part les travaux mentionnés au chapitre 4 , il n'existe guère d'ouvrages ayant pour thème la comparaison des données radar et grêlimétriques. Cette situation provient de la difficulté d'utiliser simultanément un système radar très complexe et un réseau très dense de grêlimètres pendant une saison entière, car cela demande beaucoup de temps et engage de fortes dépenses pour obtenir peu de données lors de quelques chutes de grêle.

Nos recherches sur les chutes de grêle ont utilisé, en Suisse centrale, un réseau très dense de grêlimètres qui est soigneusement surveillé, s'étend sur une superficie de $1340 \mathrm{~km}^{2}$ et comprend 335 stations au sol. La maintenance de ce réseau est assurée par une équipe d'une dizaine de personnes qui coopère à un programme international de lutte contre la grêle depuis 1977. (Federer et al. 1978). Lorsqu'un nuage dangereux se présente dans la zone en question, le réseau est immédiatement alerté.

La comparaison entre les données du système radar et celles du réseau de grêlimètres est très satisfaisante et sera développée au chapitre 4.

$\mathrm{Au}$ chapitre 5 , seront examinés les problèmes de chutes de grêle, faibles et moyennes, qui présentent des difficultés supplémentaires, à savoir : la séparation de la grêle et de la pluie, puis les énormes gradients des cellules de grêle qui sont à l'origine d'incertitudes importantes notamment en ce qui concerne les relevés du réseau de grêlimètres à larges mailles dont on dispose actuellement.

Il s'est révélé possible de comparer ces dernières données avec des mesures très denses effectuées par radar pour en déduire une évaluation de la représentativité des mesures au sol.

\section{Détection de la grêle}

Les recherches sur la grêle, en URSS, remontent à plus de 20 ans. Les différents critères en usage se distinguent par le nombre et le type des paramètres retenus. Le critère que nous employons dans notre programme de lutte contre la grêle a été développé en collaboration

LA HOUILLE BLANCHE/N ${ }^{\circ} 5 / 6-1983$ 


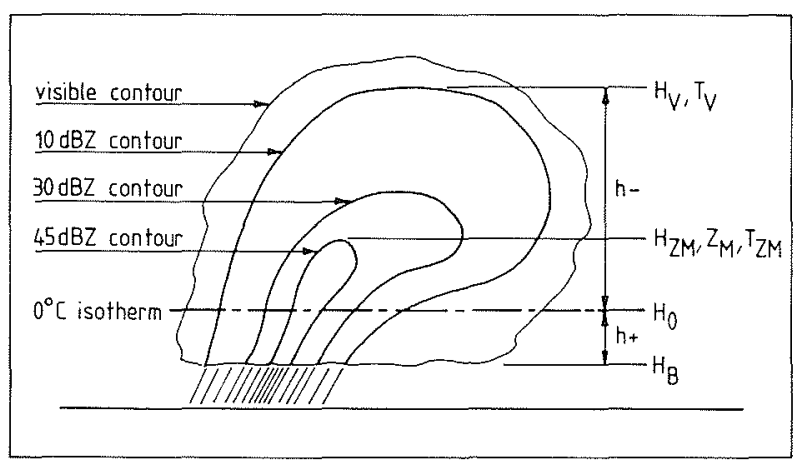

Figure 1 - Schéma d'une cellule de grêle. Les paramètres employés pour le critère sont à droite.

$$
H_{B} \text { : base du nuage, } H_{v}-H_{0}=h-, H_{0}-H_{B}=h+
$$

avec des scientifiques soviétiques, en tenant compte des particularités de notre climatologie (Federer et al. 1978).

$\mathrm{Ce}$ critère est une modification du critère Moldave et emploie les paramètres suivants (représentés sur la figure 1) :

- hauteur du sommet du contour $10 \mathrm{dBZ}$ mesuré par radar $-H_{v}$

- température correspondante au sommet du contour $10 \mathrm{dBZ}-T_{v}$;

- hauteur de la réflectivité maximale $H_{z m}$;

- relation des hauteurs entre les régions chaudes et froide du nuage $(h-/ h+)$.

Pendant toute la vie d'une cellule et à intervalles courts ( 1 ou 2 minutes), les paramètres doivent être continuellement mis à jour. Il en est de même pour toute nouvelle cellule qui apparait ou se forme. Les valeurs de ces paramètres sont ensuite transformées à l'aide de tableaux empiriques, puis combinées pour donner une probabilité complexe de grêle $P$.

$$
P=P\left(H_{v}, T_{v}, H_{z m}, T_{z m}, Z_{m}, h-/ h+\right)
$$

En pratique, cette procédure est beaucoup plus simple qu'il n'apparait car il n'y a que 3 paramètres :

$$
H_{v}-H_{z m}-Z_{m}
$$

qui doivent être mesurés continuellement, les autres ne dépendant pas des cellules individuelles et pouvant être déterminés par la structure thermodynamique de l'air ambiant. La probabilité complexe de grêle peut donc être évaluée en temps réel à l'aide d'un petit ordinateur.

On peut trouver une description détaillée des tables et du calculateur employés dans un rapport du "Cloud Physics Group ETH" (1976).

Une cellule est considérée comme grêligène lorsque les conditions suivantes sont remplies :

$$
\begin{aligned}
P & >50 \% \\
Z_{m} & >45 \mathrm{dBZ} \\
H_{z m} & >H_{0}
\end{aligned}
$$

Pour évaluer la validité de ce critère, une comparaison a été effectuée sur 195 cellules entre les données radar et les traces correspondantes sur le réseau au sol. Les résultats sont résumés dans le Tableau $\mathrm{I}$.

L'examen du Tableau I conduit à la conclusion que le critère $n^{\circ} 1$ est sûr. Toutes les cellules de grêle ont été
Tableau 1. - Les 195 cellules examinées par le critère 1 en vue

\begin{tabular}{|c|c|c|c|c|c|c|}
\hline Type de cellule & \multicolumn{2}{|c|}{ Grèle } & \multicolumn{2}{|c|}{ Critère 1} & $\begin{array}{c}\text { Nombre de } \\
\text { cellules }\end{array}$ & $\begin{array}{l}\text { Fusées dont } \\
\text { on a besoin }\end{array}$ \\
\hline $\begin{array}{l}\text { cellule de pluie } \\
\text { faible }\end{array}$ & - & $x$ & - & $x$ & 124 & 0 \\
\hline $\begin{array}{l}\text { cellule de pluie } \\
\text { vigoureuse }\end{array}$ & - & $x$ & $x$ & - & 38 & 77 \\
\hline cellule de grêle & $x$ & - & $x$ & - & 33 & 165 \\
\hline failles & $x$ & - & - & $x$ & 0 & 0 \\
\hline total & & & & & $\overline{195}$ & 242 \\
\hline
\end{tabular}
d'occurrence de grêle. Cellule de grêle signifie que le critère a été rempli et qu'on a trouvé de la grêle. Failles veut dire que le critère n'est pas rempli mais qu'on a trouvé de la grêle au sol. La dernière colonne indique le nombre de fusées hypothétique dont on aurait besoin pour inséminer les cellules. . . . . .

détectées. Cependant 38 cellules de pluie vigoureuse, n'ayant pas produit de grêle au sol, mais répondant à ce même critère, ont été définies comme grêligène (fausse alerte). Cette propriété du critère conduit une perte de $32 \%$ d'agent inseminateur, comme l'indique la dernière colonne du Tableau I. La non-proportionalité des rapports entre le nombre de cellules et le nombre de fusées tirées provient du fait que les cellules de grêle (33) exigent au moins deux fois plus de matière séminante que les cellules de pluie, parce qu'elles durent plus longtemps.

Une comparaison ultérieure entre les données radar et celles des grêlimètres, pour les 195 cellules examinées, montre que le critère $n^{\circ} 1$ peut être remplacé par un autre critère, plus simple et légèrement plus efficace, le critère $n^{\circ} 2$ :

$$
H_{45}>H_{0}+1,4 \mathrm{~km}
$$

Ce critère revient à dire que si le sommet du contour $45 \mathrm{dBZ}$ de la figure 1 se situe à plus de 1400 mètres audessus de l'isotherme $0^{\circ} \mathrm{C}$, on est très probablement en présence d'une "cellule de grêle". Le rapport entre le nombre de cellules de grêle et celui de cellules de forte pluie devient alors $33 / 28$ au lieu de $33 / 38$ (critère $n^{\circ} 1$ ) et la perte d'agent inséminateur passe de 32 à $27 \%$.

Cette découverte est un résultat important pour les recherches en cours : le critère $\mathrm{n}^{\circ} 2$ n'est pas seulement plus simple, mais plus similaire au critère original NHRE employé pour prévoir une journée de grêle dans le Colorado (Foote et Knight 1979) ou lors de tentatives d'élimination de la grêle en Afrique du Sud (Mather et al. 1976).

Ce résultat, bien que surprenant, compte-tenu d'une climatologie de grêle différente dans les deux zones, s'explique par la physique des nuages et se confirme par des mesures "in situ" (Waldvogel et al. 1979). Les mêmes résultats observés en divers endroits et par des méthodes différentes confirment la validité du critère de détection de la grêle. 


\section{Fondements de la mesure de grêle par radar}

Pour pouvoir détecter les cellules de grêle dans un ensemble de nuages, il est indispensable de reconnaitre les cellules dangereuses dès leur formation. En outre, les mesures des chutes de grêle par radar devraient donner des résultats aussi précis que possible en ce qui concerne les dommages ou la dimension des grêlons au sol. Pour cette raison, les données radar sont intégrées pendant la durée de l'orage de grêle.

La première approche qualitative de ce problème a été publiée par Geotis en 1963 et une première solution de la mesure quantitative des chutes de grêle par un radar de $10 \mathrm{~cm}$ de longueur d'onde a été présenté en 1978 par Waldvogel et al.

Les principaux problèmes à résoudre pour parvenir à une bonne estimation de la grêle sont les suivants :

1) relation entre le flux de l'énergie cinétique des grèlons

$($ E) et la réflectivité $(Z)$,

2) propriétés rétrodiffusantes des grêlons,

3) séparation des échos radars dûs à la pluie de ceux dûs à la grêle. Un exposé des solutions aux questions 1 et 3 sera fait dans les sections suivantes.

\subsection{Relations $Z$ - $\stackrel{\circ}{E}$}

Une solution, peu compliquée, pour déterminer la relation entre $E$ et $Z$ peut-être obtenue en mesurant la ditribution du volume des grêlons. L'avantage de notre méthode réside dans le fait que $E$ et $Z$ sont dérivés du même échantillon, ce qui élimine les différences de volume, de mesure, et de position que l'on rencontre en comparant les données radar avec celles des grêlimètres d'autres exemples. Les spectres des grêlons ont été mesurés au centre d'un fort orage à grêle par des véhicules munis de spectromètres pour grêlons et gouttes de pluie. L'analyse de 175 spectres de grêlons lors de 4 fortes tempètes de grêle montre une correspondance inattendue dans les 4 relations $Z$ - $\stackrel{\circ}{E}$ et conduit à la relation suivante :

$$
\stackrel{\circ}{E}=5 \times 10^{-6} \cdot Z^{0,84}
$$

où $\stackrel{\circ}{E}$ est exprimée en Joules $/ \mathrm{m}^{2}$.s et $Z$ en $\mathrm{mm}^{6} / \mathrm{m}^{3}$. Cette équation est basée sur l'hypothèse que les grêlons sont des sphères qui rétrodiffusent suivant la loi de Rayleigh.

L'équation (3) sera utilisée ultérieurement pour transformer les données radar en relevés de grélimètres et vice-versa.

\subsection{Rétrodiffusion des grêlons}

La rétrodiffusion des ondes électromagnétiques par des grêlons a été et est encore l'objet de beaucoup de recherches théoriques et empiriques. La réaction rétrodiffusante dépend de la surface sèche ou mouillée, de la structure, de la forme des grêlons, etc. On trouve une discussion de quelques-uns de ces problèmes chez Eccles (1979) et Ulbrich (1978).

Les recherches montrent que la réaction rétrodiffusante est très complexe. Même en cas de simples sphères mouillées, par exemple, la rétrodiffusion n'est pas directement dérivable de la loi de Rayleigh. De plus, on trouve des grêlons de formes et de surface très variées dans une tempëte de grêle. Pour surmonter ce problème, on part de la relation suivante (Waldvogel et al. 1978) :

$$
\int_{\text {orage }} Z(x, y, t) d x d y d t=k \int_{\text {orage }} Z_{e}(x, y, t) d x d y d t
$$

dans laquelle:

$Z_{e}$ est la réflectivité radar équivalente mesurée par radar, $Z$ le facteur de réflectivité de Rayleigh,

$k$ une constante.

En comparant les données du spectromètre de grêle avec les données radar d'une part et celles des grêlimètres avec les mesures radar d'autre part, on trouve que $k=1$, au moins pour les chutes de grêle vigoureuses, c'est-àdire que l'on peut poser :

$$
Z_{e}=Z
$$

pour des données qui sont intégrables en fonction du temps et de l'espace.

\subsection{La séparation entre la pluie et la grêle}

La séparation entre les échos de pluie et de grêle n'est pas possible, en théorie, avec un radar à une seule longueur d'onde. Cependant, on est parvenu à une solution simple pour les chutes de grêle vigoureuses en utilisant la méthode dite "cutting method". Elle néglige les réflectivités radar égales ou inférieures à $60 \mathrm{dBZ}$ considérées comme "dûes uniquement à la pluie" et estime par contre que les réflectivités supérieures à $60 \mathrm{dBZ}$ ne sont "dûes qu'à la grêle".

Il est évident que cette simplification, trouvée empiriquement, doit être interprétée dans un sens large, car il n'est pas impossible de trouver des points particuliers de réflectivité, les uns supérieurs à $60 \mathrm{dBZ}$ qui ne sont dûs qu'à la pluie, et d'autres inférieurs à $60 \mathrm{dBZ}$ issus uniquement de la grêle. Mais si l'on regarde les intégrales (4) de temps et d'espaces des chutes de grêle, cette simplification ne peut influencer la valeur moyenne.

Il existe une seconde méthode, plus perfectionnée, dite "gradual method". Elle est similaire à la "cutting method" sauf en ce qui concerne la transition entre les réflectivités "pluie" et "grêle" qui s'effectue graduellement et non d'une façon abrupte. Les réflectivités radar d'une région de pluie/grêle entre 55 et $65 \mathrm{dBZ}$ sont

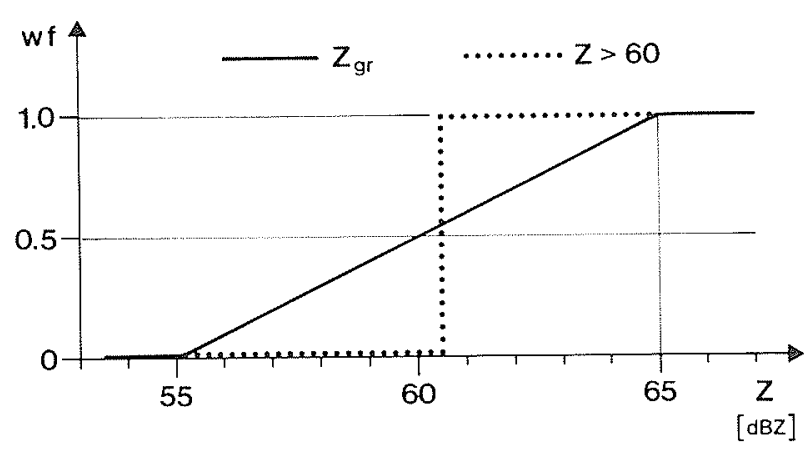

Figure 2 - Les fonctions poids pour la "cutting method" (...) et la "gradual method" (- - . La valeur du flux juste de l'énergie cinétique est reçue en multipliant la valeur $E$ de l'équation 3 avec le poids de la valeur $Z$ correspondante. 
déterminées en utilisant une fonction poids : à $56 \mathrm{dBZ}$, le poids de la contribution, d'après l'équation (3) représente $10 \%$ de sa vraie valeur, à $57 \mathrm{dBZ}$, il est de $20 \%$, à $65 \mathrm{dBZ}$ et au-delà, il est de $100 \%$.

La figure 2 illustre les deux méthodes en donnant la fonction poids pour les différentes valeurs de $Z$.

Une comparaison entre les deux méthodes montre que la différence entre les valeurs "temps" et "espace" intégrées de $E(x, y, t)$ est petite pour les chutes de grêle vigoureuses, mais la "gradual method" semble supérieure à la "cutting method" lorsqu'il s'agit de chutes de grêle, moyennes ou légères, car elle tient compte également des réflectivités inférieures.

Ce problème est actuellement en cours d'étude.

\section{Exemple de mesure de grêle}

La figure 3 montre deux diagrammes relatifs à la chute de grêle du 9 septembre 1979. Le diagramme 3 a donne les contours de la réflectivité radar pour $55 \mathrm{dBZ}$. Ces contours sont enregistrés chaque minute et repérés de 2001 à 2121 . Pendant ce laps de temps l'orage s'est déplacé d'ouest en est. La position du radar de coordonnées $(0,0)$ est marquée d'une croix sur la figure. Le diagramme $3 b$ reproduit les isolignes obtenues à partir des données radar dont l'énergie cinétique est de 1,10 et $100 \mathrm{~J} / \mathrm{m}^{2}$. La "gradual method" a été utilisée pour cette représentation et de ce fait, l'isoligne de la figure $3 \mathrm{~b}$ correspond bien avec l'enveloppe des contours de la figure 3 a pour $55 \mathrm{dBZ}$.

\subsection{Structure fine d'une chute de grêle}

La figure 4 est un exemple de correspondance entre les données radar et celles des grélimètres. Elle montre:

- en figure $4 \mathrm{a}$, la position des grêlimètres, leur identification, la valeur de l'énergie cinétique en $\mathrm{J} / \mathrm{m}^{2}$, la sur-
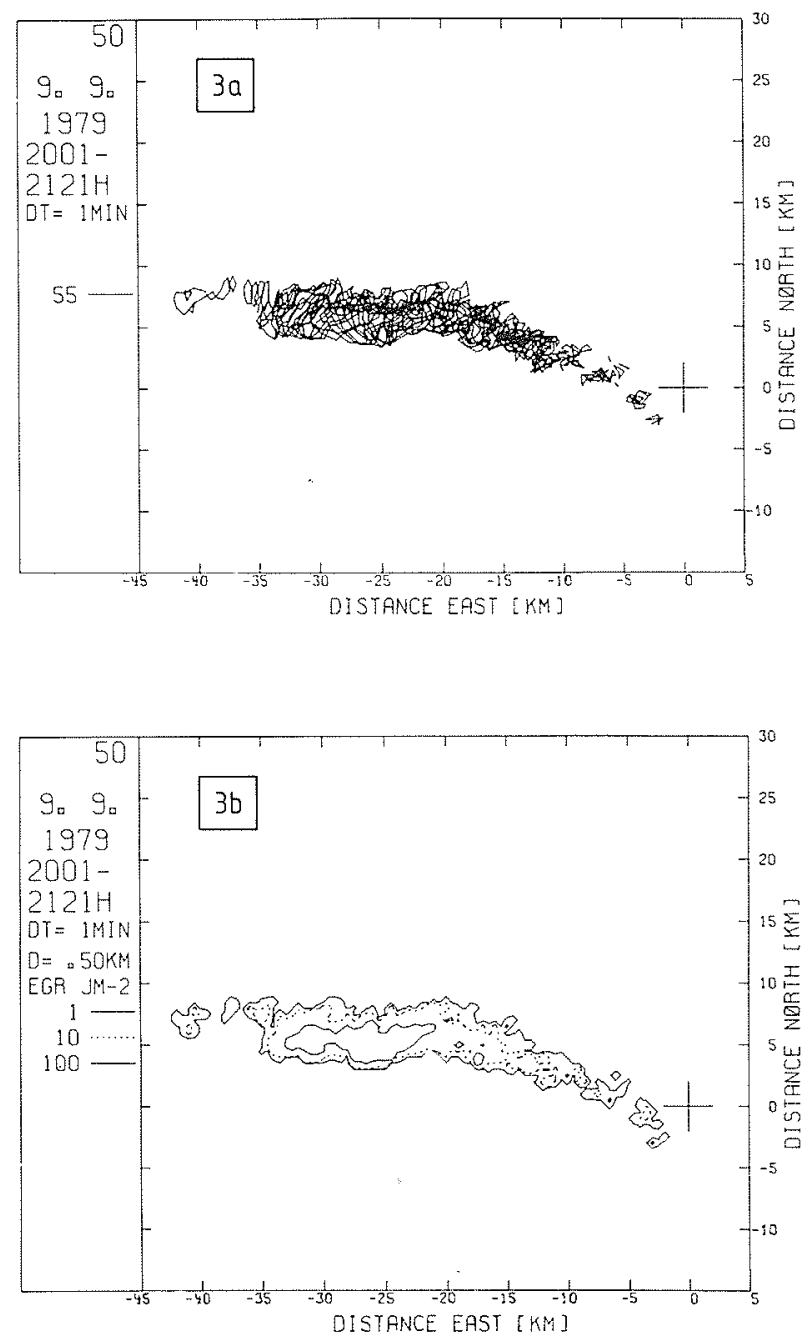

Figure 3 - Les contours de $55 \mathrm{dBZ}$ de la réflectivité radar d'une cellule de grêle du 9 septembre 1979 sont dépeints à la figure $3 \mathrm{a}$. Les contours de l'isoénergie de cette cellule pour 1,10 et 100 $\mathrm{J} / \mathrm{m}^{2}$ en utilisant la "gradual method"sont montrés à la figure $3 \mathrm{~b}$.
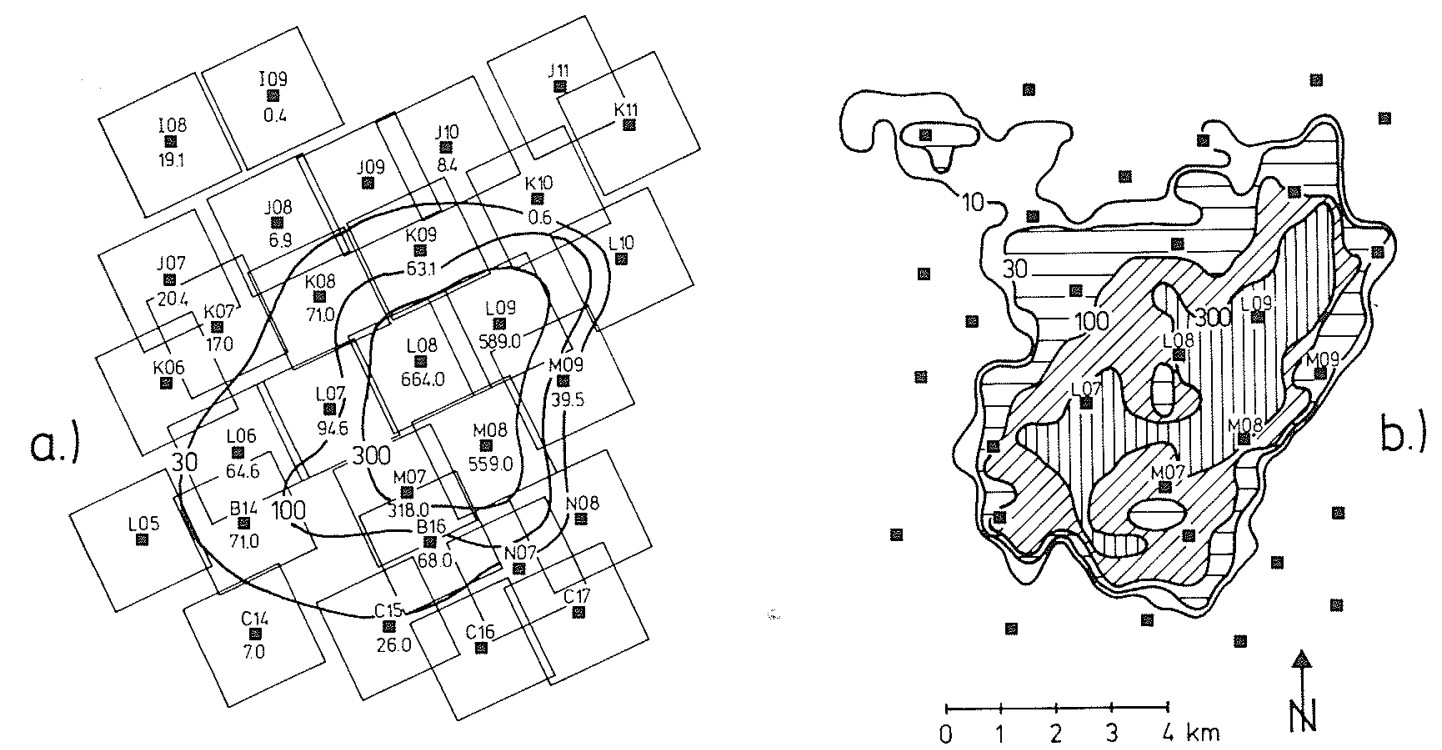

Figure 4 - La chute de grêle du $1^{\text {er }}$ juin 1978. Les plus importantes valeurs de grêlimêtre sont données en (a), en montrant les positions de grêlimètre avec les valeurs d'énergie $\left(\mathrm{J} / \mathrm{m}^{2}\right)$ et les surfaces représentées par les grêlimètres. Les isolignes dérivées du radar de $E$ ( $Z$ ) sont exprimées (b) aussi en $\left(\mathrm{J} / \mathrm{m}^{2}\right)$. On a utilisé ici la "gradual method". 
face affectée à chaque grêlimètre et les isolignes pour 300,100 et $300 \mathrm{~J} / \mathrm{m}^{2}$,

- en figure $4 \mathrm{~b}$, les isolignes dérivées des données radar dont l'énergie cinétique est de $10,30,100$ et $300 \mathrm{~J} / \mathrm{m}^{2}$.

La résolution des mesures est de $300 \mathrm{~m}$ pour les données radar et de $2 \mathrm{~km}$ pour les grêlimètres. De ce fait, la structure de la chute de grêle apparait beaucoup plus fine sur la figure $4 \mathrm{~b}$ qui montre également des écarts d'énergie avec les grêlimètres de la figure $4 \mathrm{a}$.

Les mesures par radar permettent non seulement de rechercher la structure spatiale fine d'une précipitation, mais également sa structure temporelle comme l'indique la figure 5 sur laquelle on distingue deux maximums à $18 \mathrm{~h} 56$ et $19 \mathrm{~h} \mathrm{06.} \mathrm{Ces} \mathrm{maximums} \mathrm{ont} \mathrm{:}$

- un flux d'énergie $(Z>60 \mathrm{dBZ})$ qui est respectivement de 0,50 et de $0,42 \mathrm{GJ} / \mathrm{min}$,

- une durée de 5 minutes pour l'un et de 10 minutes pour l'autre.

Ils sont séparés par un intervalle de temps de 5 minutes pendant lequel l'énergie cinétique des grêlons représente environ $50 \%$ de l'énergie maximale de la chute de grêle.

Cet intervalle de 5 minutes correspond à une phase de production de grêle forte à l'intérieur d'une chute continue de grêle plus faible.

Le flux d'énergie cinétique global, calculé suivant la "cutting method" est représenté sur la figure 5 :

- par une ligne en pointillé pour $Z>55 \mathrm{dBZ}$,

- par un trait continu pour $Z>60 \mathrm{dBZ}$.

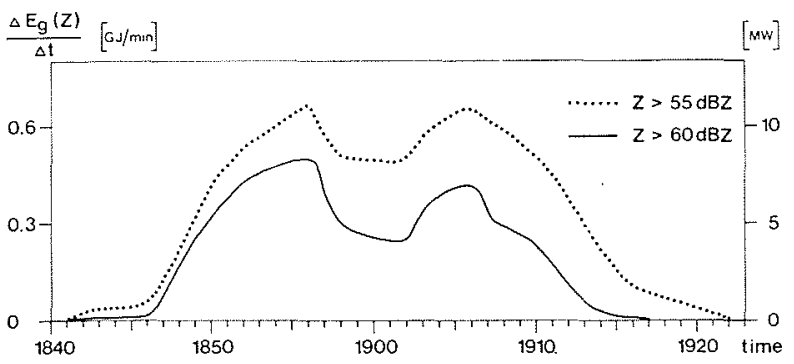

Figure 5 - Flux de l'énergie cinétique globale de la chute de grêle en fonction de temps. La "cutting method" a été employée avec un seuil à 55 et à $60 \mathrm{dBZ}$.

L'intervalle entre ces deux courbes correspond à l'intensité de la pluie pendant la chute de grêle et indique que la phase de pluie forte est plus intensive vers la fin de la phase de grêle.

En outre, le rapport :

$$
\stackrel{\circ}{E}_{g}(Z>55 \mathrm{dBZ}) / \hat{E}_{\delta}(Z>60 \mathrm{dBZ})
$$

- est constant et égal à 1,3 jusqu'à l'heure marquée 1856 ,

- est compris entre 1,5 et 2,1 au temps repéré 1907

- et devient supérieur à 3 pour les dernières minutes de la chute de grêle.

Sa valeur moyenne est de 1,8 entre les temps 1841 et 1922.

\subsection{Concordance des mesures par radar et par grêlimètres}

Il est intéressant de comparer sur le Tableau II les énergies cinétiques $E_{g}(Z)$ globales dérivées des données radar à l'aide des différentes méthodes employées. L'énergie cinétique globale est obtenue en multipliant la valeur de l'énergie cinétique totale par la surface affectée à chaque grêlimètre. Par exemple, l'énergie cinétique globale $E_{g}$ dérivée des grêlimètres a pour valeur:

$$
E_{g}=2954 \mathrm{~J} / \mathrm{m}^{2} \times 3,8 \mathrm{~km}^{2}=11,23 \mathrm{GJ}
$$

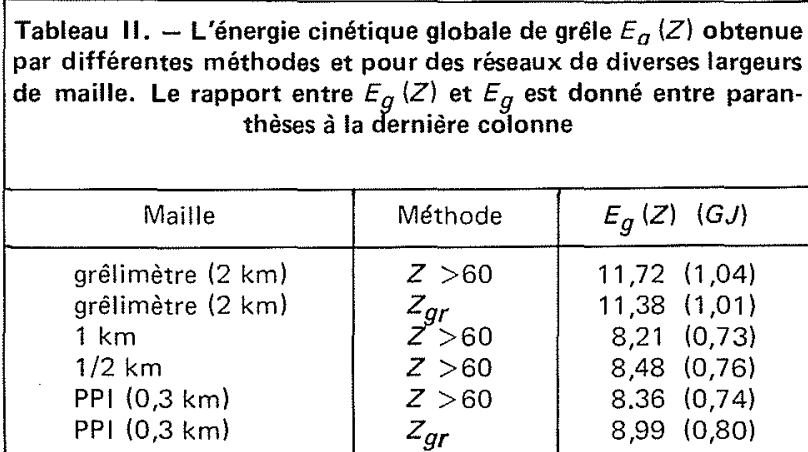

On peut facilement discerner deux groupes d'énergies cinétiques globales:

- la première, provenant des données radar mesurées aux positions des grêlimètres,

- la seconde, déduite des informations des grêlimètres au sol.

La cohérence des résultats des deux groupes d'énergie est étonnante et confirme la représentativité des mesures. La différence de $30 \%$ environ entre les deux valeurs moyennes peut s'expliquer en considérant la structure fine de la figure $4 b$, qui montre quelques positions de grêlimètres à l'intérieur d'un contour d'isoénergie plus élevé, mais proche de celui-ci. Ceci conduit à une surestimation de l'énergie cinétique globale $E_{g}$ quand on multiplie simplement chaque valeur d'un grêlimètre par la surface de $3,8 \mathrm{~km}^{2}$ qui lui est affectée. Une meilleure valeur de l'énergie cinétique globale ne peut être obtenue qu'en employant un réseau plus dense de grêlimètres ou én utilisant un système combiné de radar et de grêlimètres.

\subsection{Corrélation radar-grêlimètres}

La meilleure méthode pour obtenir une évaluation quantitative de la concordance entre les données radar et grêlimètriques est de corréler les deux séries de données.

D'abord, par simple corrélation; point par point, entre les énergies cinétiques mesurées par les grêlimètres d'une part et par radar, d'autre part, pour chacune des positions des grêlimètres dans la projection PPI. Comme il y a 38 emplacements de grêlimètres, il y a 38 paires de valeurs de l'énergie cinétique $\left[E_{i}, E_{i}(Z)\right]$ et pour chaque paire, l'une de ces valeurs, soit la mesure par grélimètre $\left(E_{i}\right)$, soit celle par radar $E_{i}(Z)$ est supérieure à zéro. La partie la plus importante des chutes de grêle étant représentée sur la figure 4 a, il n'est pas possible d'identifier 


\begin{tabular}{|c|c|c|}
\hline & $Z>60$ & $Z_{g r}$ \\
\hline$r(38)$ & 0,84 & 0,85 \\
\hline $\begin{array}{cc}\Sigma E_{j}(Z) / & \Sigma E_{i} \\
\text { grêli- } & \text { grêli- } \\
\text { mètres } & \text { mètres }\end{array}$ & 1,04 & 1,01 \\
\hline
\end{tabular}

tous les grêlimètres touchés par des grêlons (voir Waldvogel et al. 1980 pour plus de détails) et l'une au moins de ces 38 valeurs $E_{i}$ est égale à zéro.

Quant au radar, les valeurs $E_{i}(Z)$ sont différentes et l'on en trouve 26 égales à zéro avec la "cutting method" et 18 avec la "gradual method". Pourtant la différence entre les deux méthodes est petite, comme l'indique le Tableau III qui donne les coefficients de corrélation $r$ et le rapport entre les énergies cinétiques mesurées par radar et par grélimètres.

Ce résultat confirme le fait que quelques grêlimètres seulement contribuent réellement à la mesure de l'énergie cinétique totale et pour cette raison on trouve des coefficients de corrélation élevés malgré les différentes méthodes utilisées.

Par la suite, on continuera à rechercher la corrélation entre les mesures par radar et par grêlimètres, cependant les mesures par radar ne seront plus effectuées depuis la position des grêlimètres mais à partir d'un point légèrement déplacé comme l'indique la figure 6 .

La valeur $E_{i}(Z)$ est dérivée des mesures effectuées par radar en un endroit de coordonnées $\left(x_{i}+\Delta x\right.$ et $\left.y_{i}+\Delta y\right)$ avec $\left(x_{i}, y_{i}\right)$ correspondant à l'emplacement du $\mathrm{n}^{\mathrm{i} e ̀ m e}$ grêlimètre et $(\Delta x, \Delta y)$ au vecteur de déplacement.

Ainsi chaque point de mesure par radar est déplacé du même vecteur par rapport à la position du grêlimètre correspondant.

En corrélant les valeurs $E_{i}(Z, \Delta x, \Delta y)$ avec $E_{i}$, on trouve un coefficient de corrélation $r^{\prime}=r(\Delta x, \Delta y)$ qui est fonction du vecteur de déplacement.
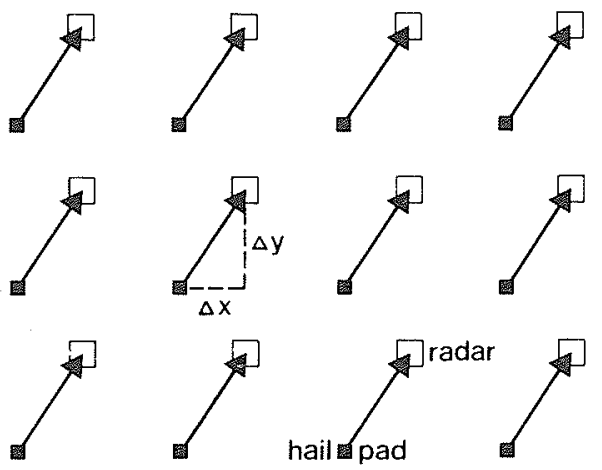

Figure 6 - Un tableau schématique de la dislocation des volumes radar illuminés à l'égard des positions de grêlimètre correspondantes. Ainsi on trouve un coefficient de corrélation dépendant du vecteur de dislocation $(\Delta x, \Delta y)$.

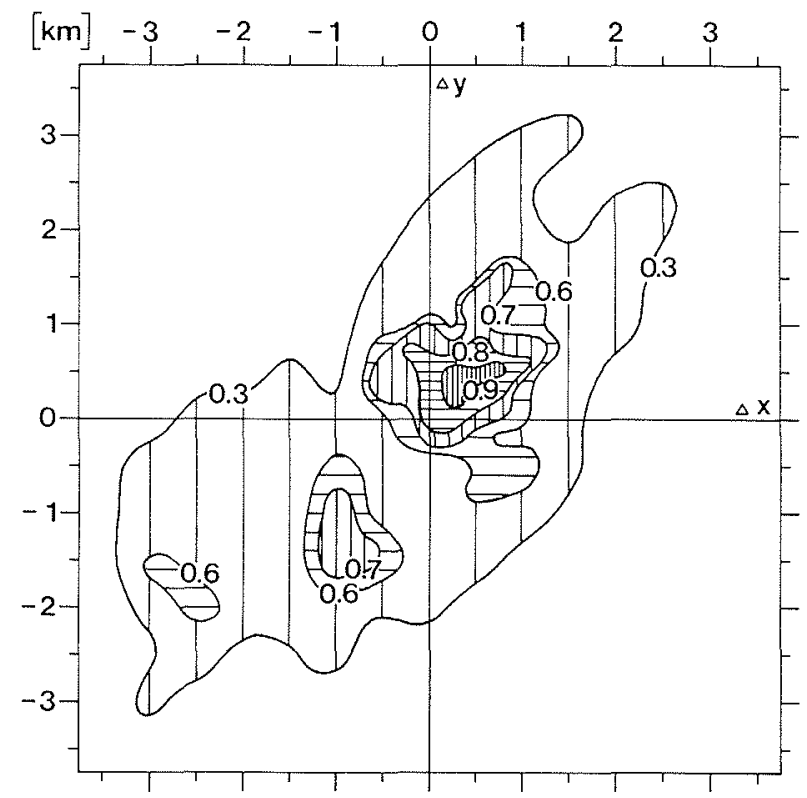

Figure 7 - Les isolignes du coefficient de corrélation $r$ entre les données radar et celles de grêlimètre sur la superficie du vecteur de dislocation $(\Delta x, \Delta y)$.

Les résultats de ces calculs sont reportés sur la figure 7. Le coefficient de corrélation $r(0,0)=0,84$ déjà mentionné dans le Tableau III ne correspond pas à une valeur maximale, car un coefficient $r=0,94$ a été trouvé après déplacement en direction N.E. de tous les points de mesure par radar d'un vecteur $(1 / 3 \mathrm{~km}, 1 / 3 \mathrm{~km})$ opposé à la direction moyenne de la chute de grêle.

Ce résultat est confirmé par une estimation de la vélocité moyenne de chute des grêlons à $20 \mathrm{~m} / \mathrm{s}$.

En supposant :

- que les grêlons tombent d'une altitude de $1,5 \mathrm{~km}$, - que la longueur du vecteur de déplacement soit de $0,47 \mathrm{~km}$,

- que la vitesse moyenne du déplacement de l'orage soit de $20 \mathrm{~km} / \mathrm{h}$,

le calcul démontre que la vitesse moyenne de chute des grêlons est de l'ordre de $18 \mathrm{~m} / \mathrm{s}$, ce qui correspond à notre estimation de $20 \mathrm{~m} / \mathrm{s}$.

\section{De la représentativité des mesures au sol}

Les remarques du chapitre 4 , relatives à la concordance et à la corrélation des énergies cinétiques d'une chute de grêle entre les mesures effectuées avec un radar de $10 \mathrm{~cm}$ de longueur d'onde et celles obtenues à l'aide des grêlimètres, ne concernent que les chutes de grêle importantes observées dans des conditions "idéales", à savoir que :

- le radar est près de la chute de grêle (distance maximale $20 \mathrm{~km}$ ),

- le nuage observé est près du sol (moins de $1500 \mathrm{~m}$ ), - le volume testé par le radar est petit (moins de 1/30 $\left.\mathrm{km}^{3}\right)$.

S'il s'agit de chutes de grêle, petites ou moyennes, qui ne sont pas nécessairement à proximité du radar, on est 
en présence de difficultés supplémentaires dont les plus importantes sont relatives :

a) à une modification de la relation $(Z, \stackrel{\circ}{E})$,

b) à un nouveau schéma de séparation entre les réflectivités radar dues à la pluie et celles dûes à la grêle,

c) à la fonte des grêlons au cours de leur chute,

d) à la représentativité des mesures des grélimètres ("vérité au sol"');

L'examen des points a), b) et c) consiste en une comparaison entre les mesures faites par grêlimètres et les données radar correspondantes. Quant au point d) concernant la "vérité au sol", la précision dont on dispose est aléatoire au cours d'une chute de grêle car quelquesuns, voire à la limite tous les grêlimètres, peuvent ne pas avoir reçu un seul grêlon. Ce point est difficile et très important. C'est la raison pour laquelle cet aspect du problème a été traité théoriquement et expérimentalement de manière différente. Jusqu'à présent aucune réponse valable en général n'a été proposée en ce qui concerne la représentativité des mesures par grêlimètres.

Dans un travail récent, Long (1980), a discuté de la précision des estimations de la fréquence de grêle en un point et de la surface couverte par ces précipitations à l'intérieur d'une région, en utilisant les concepts statistiques et probabilistiques. Une relation assez simple fut trouvée entre le nombre de positions observant la grêle et la précision des différentes estimations. Dans un travail antérieur, Long avait examiné la précision des estimations de la masse et de l'énergie cinétique de la grêle lors de différentes chutes en fonction de la maille du réseau.

Le but de ce chapitre est de parvenir à une estimation de la précision de l'énergie cinétique des chutes de grêle en fonction de la densité du réseau de grélimètres et de l'énergie cinétique de la chute de grêle elle-même. Pour cette raison, les valeurs dérivées des données radar sur 202 cellules de grêle ont été utilisées pour simuler des mesures de chute de grêle par réseau. A l'aide de cette méthode, il devient possible de construire des réseaux de grêlimètres de différentes densités. En conséquence, les mesures réelles des grêlimètres peuvent être prises en considération afin de les comparer avec les données radar.

\subsection{La méthode}

Un réseau hypothétique de grêlimètres $(1 / 2 \mathrm{~km}, 1 / 2$ $\mathrm{km})$ peut être défini pour évaluer les différentes énergies cinétiques globales en prenant les éléments du PPI qui sont autour du radar avec une maille de $1 / 2 \mathrm{~km}$. Chaque grêlimètre représente $1 / 4 \mathrm{~km}^{2}$. A l'aide de ce réseau régulier $(1 / 2,1 / 2)$, on peut construire des réseaux moins denses, par exemple : 4 réseaux $(1,1), 16$ réseaux $(2,2), 36$ réseaux $(3,3)$ et ainsi de suite.

Les énergies cinétiques globales dérivées de tels réseaux peuvent être utilisées pour estimer les écarts type des énergies cinétiques globales des réseaux hypothétiques de grêlimètres de différentes mailles.

\subsection{Les résultats}

Les résultats de la méthode décrite ci-dessus sont reportés sur la figure 8 . Pour chacune des 202 cellules de grêle observées à partir d'un réseau de grélimètres dont la "largeur de maille" est de $4 \mathrm{~km}$, on obtient, pour

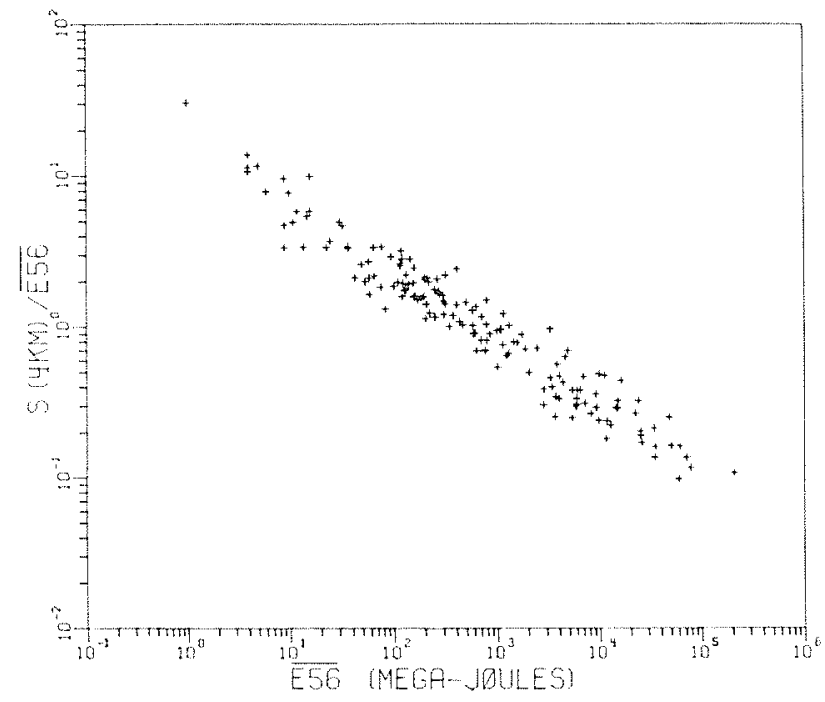

Figure 8 - Ecarts types normalisés $s(4 \mathrm{~km}) / \overline{\mathrm{E} 56}$ contre les valeurs moyennes $\overline{\mathrm{E} 56}$ d'un réseau de $4 \mathrm{~km}$. Le coefficient de corrélation et la ligne de regression sont indiqués dans le Tableau IV.

\begin{tabular}{|c|c|c|c|c|c|c|c|}
\hline Méthode & Maille & $\begin{array}{l}\text { Coefficient de } \\
\text { correlation } r\end{array}$ & $\begin{array}{r}\text { Ligne } \\
\log (s / \bar{E})\end{array}$ & $a_{1} \log (\bar{E})$ & Eca & normalisé & $/ \bar{E}$ \\
\hline \multirow[b]{2}{*}{$E 56$} & & & $a_{0}$ & $a_{1}$ & $\bar{E}=10^{2}$ & $\bar{E}=10^{3}$ & $\bar{E}=10^{4}$ \\
\hline & $\begin{array}{l}s(1 \mathrm{~km}) \\
s(2 \mathrm{~km}) \\
s(4 \mathrm{~km})\end{array}$ & $\begin{array}{l}0,93 \\
0,97 \\
0,97\end{array}$ & $\begin{array}{l}0,642 \\
0,955 \\
1,233\end{array}$ & $\begin{array}{l}-0,534 \\
-0,479 \\
-0,436\end{array}$ & $\begin{array}{r}37,5 \% \\
99,3 \% \\
229,6 \%\end{array}$ & $\begin{array}{l}11,0 \% \\
33,0 \% \\
84,1 \%\end{array}$ & $\begin{array}{r}3,2 \% \\
10,9 \% \\
30,8 \%\end{array}$ \\
\hline E61 & $\begin{array}{l}s(1 \mathrm{~km}) \\
s(2 \mathrm{~km}) \\
s(4 \mathrm{~km})\end{array}$ & $\begin{array}{l}0,95 \\
0,97 \\
0,97\end{array}$ & $\begin{array}{l}0,882 \\
1,176 \\
1,445\end{array}$ & $\begin{array}{l}-0,546 \\
-0,488 \\
-0,454\end{array}$ & $\begin{array}{r}61,7 \% \\
158,5 \% \\
345,1 \%\end{array}$ & $\begin{array}{r}17,5 \% \\
51,5 \% \\
121,3 \%\end{array}$ & $\begin{array}{r}5,0 \% \\
16,7 \% \\
42,7 \%\end{array}$ \\
\hline EGR & $\begin{array}{l}s(1 \mathrm{~km}) \\
s(2 \mathrm{~km}) \\
s(4 \mathrm{~km})\end{array}$ & $\begin{array}{l}0,92 \\
0,94 \\
0,93\end{array}$ & $\begin{array}{l}0,183 \\
0,521 \\
0,825\end{array}$ & $\begin{array}{l}-0,398 \\
-0,351 \\
-0,314\end{array}$ & $\begin{array}{r}24,4 \% \\
65,9 \% \\
157,4 \%\end{array}$ & $\begin{array}{r}9,7 \% \\
29,4 \% \\
76,4 \%\end{array}$ & $\begin{array}{r}3,9 \% \\
13,1 \% \\
37,1 \%\end{array}$ \\
\hline
\end{tabular}


chaque cellule, 64 valeurs d'énergie cinétique globale $\left(E_{56,4}\right)$ dont on peut calculer la valeur moyenne $\overline{E_{56}}$ et l'écart type $s(4 \mathrm{~km})$.

Les 202 valeurs des écarts type normalisés $s(4 \mathrm{~km}) /$ $E_{56}$ sont corrélées avec les valeurs moyennes $\overline{E_{56}}$. Une bonne corrélation et une diminution de l'écart type normalisé correspondent à une énergie cinétique globale croissante. L'écart type normalisé pour un réseau de $4 \mathrm{~km}$ est de 6,3 pour une énergie de $10 \mathrm{MJ}$.

Les résultats des trois méthodes différentes pour dériver les énergies cinétiques globales sont rassemblés dans le tableau IV où sont également mentionnés les coefficients de corrélation, les lignes de régression et les valeurs des écarts types normalisés pour les 3 valeurs caractéristiques de l'énergie cinétique globale : 0,$1 ; 1$ et $10 \mathrm{GJ}$.

Les coefficients de corrélation reportés sur la figure 8 se retrouvent dans d'autres séries de données. La pente des lignes de régression qui est de 0,5 pour $E_{56}$ et $E_{61}$ signifie que la valeur de l'écart type normalisé diminue avec la racine carrée de l'énergie cinétique globale. Ce résultat confirme les travaux de Long (1980).

Une remarque importante doit cependant être faite au sujet de la généralisation des résultats obtenus à l'aide des réseaux de grêlimètres. En raison de la surface relativement étendue testée par le radar $\left(1 / 10-1 / 3 \mathrm{~km}^{2}\right)$, les résultats dérivés de ces données doivent être considérés comme une limite inférieure d'incertitude et celle-ci prise en compte lorsqu'il s'agit des énergies cinétiques globales ou des paramètres de chute de grêle dérivés des réseaux. En conséquence, les énergies dérivées des informations des grêlimètres ont un degré d'incertitude de l'ordre de celui qui est indiqué dans le Tableau IV.

\section{Relations entre la surface, la durée de vie et l'énergie cinétique des cellules de grêle}

L'influence de la margeur de la maille d'un réseau de grêlimètres sur la précision de l'énergie cinétique des cellules de grêle peut être exprimée d'une manière satis.

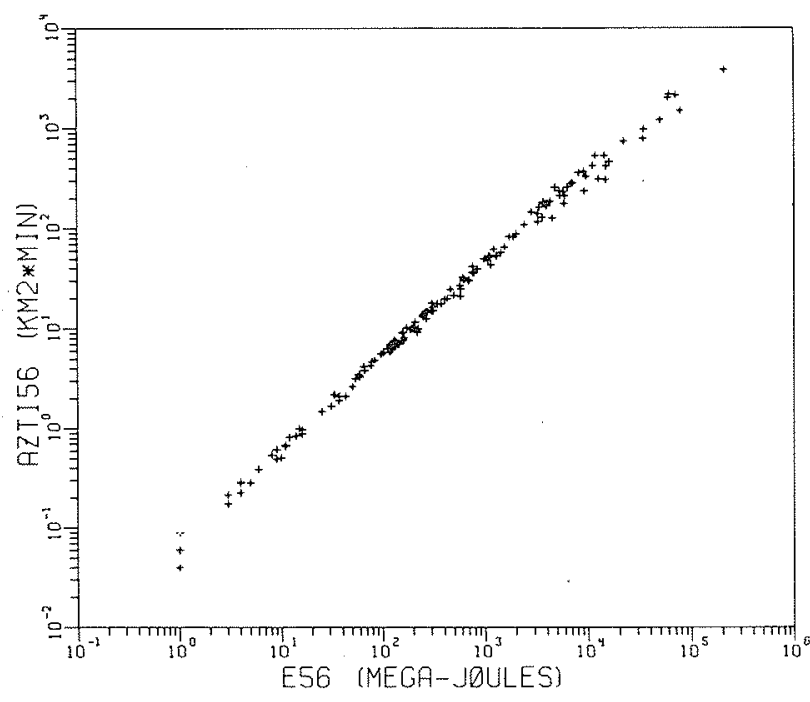

Figure 9 - Les surfaces des contours $56 \mathrm{~dB}(Z)$ in tégrées en temps (AZTI 56) contre E56. Le coefficient de corrélation est de 0.998 . faisante en utilisant seulement deux paramètres : la largeur de la maille et l'énergie cinétique. Pourtant, comme la surface et la durée de vie d'une cellule de grêle sont des paramètres beaucoup plus évidents que l'énergie cinétique elle-même, on considèrera la relation entre ces différents paramètres.

La surface d'une cellule de grêle ne restant pas constante pendant sa durée de vie (Fig. 3 a), le temps de parcours d'un contour de réflectivité donné et défini par Waldvogel et al. (1978b) sera utilisé dans cette recherche plutôt que la surface elle-même. La figure 9 montre la corrélation entre le symbole AZTI 56 (Area of the $56 \mathrm{~dB}$ $(Z)$ contour Time Integrated) et l'énergie $E_{56}$ pour les cellules de grêle. Une corrélation très élevée entre ces deux paramètres signifie qu'une chute de grêle de forte énergie cinétique est produite par une cellule de grêle géométriquement importante. Ce fait n'est pas surprenant en lui-même et a été employé à bon escient pour préciser que l'expression "chute de grêle forte" signifie également "chute de grêle de forte énergie cinétique". Pourtant, la valeur élevée du coefficient de corrélation de AZTI56 et de $E_{56}$ qui est de 0,998 est inattendue et peut s'expliquer par la valeur de AZTI pour les différentes réflectivités radar d'une cellule de grêle.

Une autre corrélation intéressante est représentée sur la figure 10 où les valeurs de $E_{56}$ sont corrélées avec la durée des contours de $56 \mathrm{dBZ}$. Comparée la figure 9 , on constate que la dispersion autour des points de la ligne de régression est beaucoup plus importante, le coefficient de corrélation n'étant que de 0,920 .

A cause de la grande dispersion des points autour de la ligne de régression $( \pm 5 \mathrm{~dB})$, il n'est pas possible de déterminer avec précision la valeur de $E_{56}$ et la durée $\mathrm{du}$ contour. Cependant, une bonne approximation peut être obtenue par la relation suivante :

$$
E_{56}=0,89 t^{2,033}
$$

où $t$ est la durée de parcours d'un contour (exprimée en minutes. Ce paramètre peut facilement être mesuré en temps réel) et $E_{56}$, l'énergie cinétique exprimée en MJ (méga-joules).

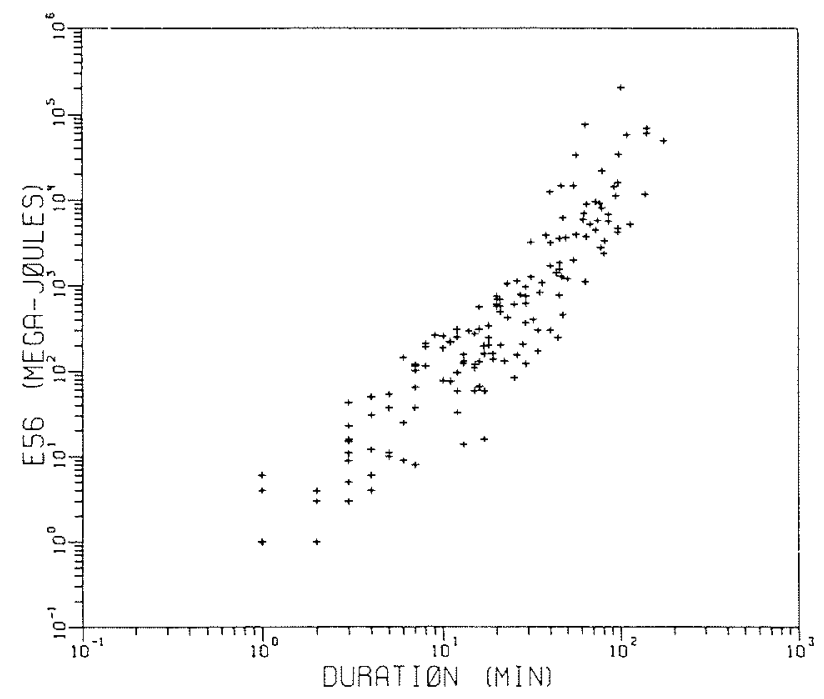

Figure 10 - L'énergie cinétique globale E 56 corrélée avec la durée des contours $56 \mathrm{~dB}(Z)$. Le coefficient de corrélation est de 0.920 . 


\begin{tabular}{|c|c|c|}
\hline \multicolumn{3}{|c|}{$\begin{array}{l}\text { Tableau } V . \text { - Les coefficients de corrélation et les relations déri- } \\
\text { vées des lignes de régression de différents paramètres d'une cel- } \\
\text { lule de grêle. La durée du contour de } 56 \mathrm{dBZ} \text { est symbolisée par } \\
\text { t et mesurée en minutes. AZTI56 est en } \mathrm{km} ? \text { min, et E56 en MJ. }\end{array}$} \\
\hline Paramètres & $\begin{array}{l}\text { Coeff. } \\
\text { de cor- } \\
\text { relation }\end{array}$ & Relation \\
\hline (AZTI56)-(E56) & 0,998 & AZTI56 $=0,077(E 56)^{0,919}$ \\
\hline$(E 56)-(t)$ & 0,920 & $E 56=0,89 \quad(t)^{2,033}$ \\
\hline$(A Z T I 56)-(t)$ & 0,929 & $A Z T I 56=0,068(t)^{1,878}$ \\
\hline$(A Z T / 56 / t)-(t)$ & 0,762 & $A Z T 156 / t=0,068(t)^{0,878}$ \\
\hline
\end{tabular}

Le Tableau $V$ rassemble les différentes corrélations définies précédemment ainsi que les deux suivantes:

- AZTI56 avec la durée de parcours d'un contour, - la surface moyenne d'une cellule avec la durée de parcours d'un contour.

La dernière corrélation donne un coefficient assez faible $(0,762)$ et comme l'équation (6), elle ne peut être utilisée pour déterminer avec précision un paramètre connaissant l'autre.

Les relations du Tableau $\mathrm{V}$ donnent au moins une idée de la surface ou de la durée de vie d'une cellule de grêle pour une énergie cinétique donnée ou vice-versa.

\section{Conclusions}

Compte-tenu des études mentionnées ci-dessus, on peut tirer les conclusions suivantes :

\subsection{Détection de la grêle}

1-a) Les cellules de grêle peuvent être détectées dès le premier stade de leur formation même avec un radar simple de $3 \mathrm{~cm}$ de longueur d'onde,

1-b) Le critère soviétique (6 paramètres) peut être réduit et ramené à un critère à un seul paramètre :

$$
H_{45}>H_{0}+1,4 \mathrm{~km}
$$

1-c) environ $50 \%$ de cellules détectées comme "cellules de grêle" ne produisent jamais de grêlons au sol. Ceci correspond à une perte d'environ $30 \%$ de l'agent inséminateur ou à une erreur sur le temps d'alerte de l'ordre de $30 \%$.

1-d) Les différents critères simples relevés en différents points du globe se ressemblent beaucoup.

\subsection{Mesure de la grêle}

2-a) on peut mesurer des chutes de grêle vigoureuses avec un radar simple de $10 \mathrm{~cm}$.

2-b) il existe une relation généralement valable entre l'énergie cinétique des grêlons $\stackrel{E}{E}$ et la réflectivité $Z$ :

$$
\stackrel{\circ}{E}=5.10^{-6} \times Z^{0,84}
$$

$2-c)$ l'ensemble des grêlons pendant une chute de grêle vigoureuse peut-être traité comme des particules rétrodiffusant suivant la loi de Rayleigh.

2-d) les réflectivités radar dûes à la pluie ou à la grêle peuvent être séparées par une méthode simple lorsqu'il s'agit de fortes chutes de grêle dans le temps et/ou en surface.

2-e) la concordance entre les paramètres de chute de grêle dérivés des données obtenues par radar ou par grêlimètre est de $30 \%$ s'il s'agit de chutes de grêle importantes.

Le coefficient de corrélation entre les énergies cinétiques des données par grêlimètre et de leurs valeurs correspondantes obtenues par radar peut atteindre 0,9 pour des chutes de grêle vigoureuses avec au moins 20 points de valeur non nulle.

\subsection{De la représentativité des mesures au sol}

3-a) l'écart type normalisé diminue lorsque l'énergie cinétique globale augmente,

3-b) cette diminution de l'écart type est proportionnelle à la racine carrée de l'énergie cinétique globale lorsque les énergies dérivées des données radar sont obtenues par la "cutting method",

3-c) l'écart type normalisé de l'énergie cinétique globale est d'environ $10 \%$ pour des chutes de grêle importantes (10 GJ) mesurées par des réseaux de grélimètres de $2 \mathrm{~km}$ de largeur de maille,

3-d) des chutes de grêle dont l'énergie cinétique est inférieure à $1000 \mathrm{MJ}$ conduisent à des écarts types aussi grands que les valeurs elles-mêmes,

3-e) la valeur des écarts types mentionnée ci-dessus représente la limite inférieure des résultats obtenus à l'aide de grêlimètres.

\section{Références}

ATLAS D. and LUDLAM F.H., (1961). -- Multi-wavelength radar reflectivity of hailstorms, J. Appl. Meteor., 2, 270-275.

BARGE B.L., (1972). - Hail detection with a polarization diversity radar, Stormy Weather Group Scientific Rep. MW-71, McGill Univ., Montreal, Canada.

Cloud Physics Group ETH, (1976). - Projekt Grossversuch IV, Bericht über 1975, Wiss. Mitteilung Nr. 75 der Eidg. Hagel Kommission, ETH Zürich.

ECCLES P.J., (1979). - Comparisons of Remote Measurements by Single - and Dual - Wavelength Meteorological Radars", IEEE Trans. Geosciences Electronics, GEA, 205-217.

FEDERER B., WALDVOGEL A., SCHMID W., HAMPEL F., ROSINI E., VENTO D., ADMIRAT P. and MEZEIX J.F., (1978). - Plan for the Swiss Randomized Hail Suppression Experiment. Design of Grossversuch IV, Pure and Appl. Geophysics, 117, 548-571.

FOOTE B.G. and KNIGHT C.A., (1979). - Results of a randomized hail suppression experiment in northeast Colorado. Part I, J. Appl. Meteor., 18, 1526-1537.

GEOTIS S.G., (1963). - Some radar measurements of hailstorms. J. Appl. Meteor., 2, 270-275.

LONG A.B., (1978). - Design of hail measurement network. Atmos. Ocean., 16, 35-48.

LONG A.B., (1980). - On estimating hail frequency and hailfall area. J. Appl. Meteor., 19, 1351-1362.

MATHER G.H. TREDDENICK D. and PARSONS R., (1976). An observed relationship between the height of the $45 \mathrm{dBZ}$ contours in storm profiles and surface hail reports, J. AppL Meteor., 15, 1336-1340. 
RINEHART R.E, and TUTTLE J.D., (1982). - Antenna beam patterns and dual-wavelength processing. J. Appl Meteor., 21, 1865-1880.

Seliga T.A. and BRINGI V.N., (1976). - Potential Use of Radar Differential Reflectivity Measurements at Orthogonal Polarizations for Measuring Precipitation. J. Appl. Meteor., 15, 69-75.

ULBRICH C.W., (1978). - Relationships of Equivalent Reflectivity Factor to the Vertical Fluxes of Mass and kinetic Energy of Hail, J. Appl Meteor., 17, 1803-1808.

WALDVOGEL A., SCHMID W. and FEDERER B., (1978a). The kinetic Energy of Hailfalls. Part I: Hailstone Spectra, J. Appl. Meteor., 17, 1515-1520.
WALDVOGER A., FEDERER B., SCHMID W. and MEZEIX J.F., (1978b). - The kinetic Energy of Hailfalls. Part II : Radar and Hailpads, J. Appl. Meteor., 17, 1680-1693.

WALDVOGEL A., FEDERER B. and GRIMM P., (1979). - Criteria for the Detection of Hail Cells, J. Appl. Meteor., 18, $1521-1525$.

WALDVOGEL A., FEDERER B. and HOGL D., (1980). - On the Correlation between Hailpad and Radar Data, Preprints 19 th Conf. Radar Meteo. AMS, Boston 1980.

WALDVOGEL A. and SCHMID W., (1982). - The kinetic energy of hailfalls. Part III : Sampling errors inferred from radar data. J. Appl. Meteor, 21, 1228-1238.

\section{Discussion}

Président : R. HLAVEK

\begin{abstract}
Le Président. - Je remercie M. WALDVOGEL qui nous a fait un exposé magistral à une vitesse éclair. Il a fait le tour de la question de la grêlimétrie par radar de façon merveilleuse. Il nous a démontré que le sérieux des Suisses et l'exactitude des montres suisses n'étaient pas une légende. Je crois que cela suppose un travail au sol considérable. Les résultats que vous donnez sont très simples, comme par exemple lat hauteur de $45 \mathrm{DBZ}$ par rapport à l'osotherme 0 . S'il est certain que vous ayiez bénéficié des travaux soviétiques, il semble que vous ayiez aussi disposé d'un réseau de mesures au sol important. C'était un exposé tout à fait exceptionnel.

Je vais ouvrir la discussion, car je pense que certaines personnes de l'assistance seront intéressées par la grêle. Jusqu'à présent, nous avons travaillé surtout sur la pluie, mais le problème de la grêle vient d'être très bien exposé, et je souhaite qu'il y ait sur ce sujet une discussion de la part des spécialistes.

M. FLOCH. - Je suppose que, pendant cette expérience, les mesures des températures aux différents niveaux, pour ce qui concerne les critères de probabilité de grêle, ont été faites par sondages sur place. Pour les usagers intéressés par la prévision de grêle, comment faire pour avoir ces températures ? Peut-on se contenter d'un radiosondage ou faut-il vraiment des mesures sous nuages, dans le nuage précipitant?
\end{abstract}

M. WALDVOGEL. - C'est un point important. On a fait un radiosondage à environ une centaine de kilomètres à l'ouest de la région d'étude; en second lieu, on a fait un radiosondage sur place, et on a comparé les deux; on n'a pas trouvé de très grandes différences. Il est certain que, dans les nuages, on a d'autres températures. Si l'on observe des cellules de nuages, on voit que la grêle se développe très rapidement; cela veut dire que le critère est rempli une demi-minute ou une minute plus tard ou plus tôt; c'est la seule différence. Ce n'est pas très grave.

M. FLOCH. - Que pensez-vous du phénomène de "pompage" lorsqu'une cellule est très active? Selon la petite expérience que j'en ai, les précipitations de grêle deviennent très violentes pendant un moment, puis cela s'estompe un peu, puis cela repart un peu plus loin et le phénomène se propage. Qu'en pensez-yous?

M. WALDVOGEL. - Oui, nous l'avons remarqué aussi. On a un maximum pendant à peu près 5 minutes; après, on a une diminution des énergies de $50 \%$ ou $30 \%$ de la valeur maximale; puis on a encore une fois une augmentation. Ces variations des chutes de grêle sont dues à la structure dynamique d'un orage en multicellules que l'on peut voir au sol.

M. GILET: - Pour la vérification du critère soviétique de sous-pression de la grêle, avez-vous pris en compte, d'une façon ou d'une autre, les données radar dont vous disposiez?

M. WALDVOGEL. - Oui, on a établi séparément les valeurs du critère d'une part à partir des données des grêlimètres, d'autre part à partir des caractéristiques des cellules détectées par rada (inséminées ou non). Les deux approches ont fourni des résultats équivalents.

M. PIRCHER. - Avez-vous trouvé d'autres critères météo non pas pour prévoir de la grêle, mais que vous puissiez associer à la présence de grêle dans un gros orage. Avez-vous beaucoup de cas de gros orages sans grêle, de gros orages avec grêle?

M. WALDVOGEL. - Pour cette expérience, qui a commencé en 1975, 50 personnes ont travaillé dans la région et on a eu une prévision assez bonne. Les observations commençaient dès $9 \mathrm{~h}$ du matin et on regardait si c'était une journée d'alarme ou non.

Avec la Météorologie Nationale Suisse, nous avons amélioré ce critère de prévision de grêle et on a maintenant de nombreux points; on peut dire qu'à présent la prévision des orages dans la région centrale est assez bonne.

On a naturellement des paramètres simples prédicteurs météorologiques, des sondages, etc... On a trouvé que la probabilité de grêle avec ces prédicteurs est toujours estimée avec des corrélations entre 0,6 et 0,7 .

M. $O B L E D$. - Si vous permettez que l'on revienne à la pluie, est-ce que, en tant que représentant suisse, vous pourriez nous faire un petit résumé de ce qui se fait en Suisse au point de vue de l'application hydrologique des radars, et, en particulier, des problèmes qui vont être rencontrés dans un environnement montagneux, qui doit être fertile en échos de sol.

M. WALDVOGEL. - Nous avons deux radars (ce ne sont pas des radars utilisant des données avec la grêle) de $5 \mathrm{~cm}$ qui couvrent toute la Suisse. On a intégré les données des deux radars. IIs explorent différentes élévations pendant un cycle de 5 à 10 minutes. On mesure le point d'intensité maximum à une certaine altitude. Dans la région observée, on a des montagnes. On ne peut mesurer qu'au dessus d'une certaine élevation dans les régions où l'on a des montagnes.

M. GILET. - Il y a actuellement à la Météorologie Française deux radars qui explorent plusieurs élevations, dont un dans la région d'Agen. Ce dernier radar a été placé là pour une expérience de climatologie de la grêle; on craint que la mécanique de ce radar tombe en panne d'un jour à l'autre, car il s'agit d'un matériel bon marché, le radar Rodin, dont la mécanique est très rudimentaire.

Des radars plus anciens, Mélodi, achetés selon d'autres critères, peuvent très bien balayer systématiquement plusieurs élevations sans danger pour leur mécanique. C'est le cas du radar de Dammartin-en-Goële, oư on fait de l'enregistrement des données en vue d'analyses ultérieures. Un fonctionnement opérationnel permanent à plusieurs élevations demande encore deux choses. Il faut d'abord un système informatique relativement conséquent; ceci suppose un financement non négligeable. En second lieu, le fonctionnement doit être en pratique totalement automatisé et cela suppose que certaines opérations soient interdites; il y a certains radars dont les responsables estiment qu'il est souhaitable de faire, par exemple, du balayage vertical de temps en temps pour déterminer l'altitude des nuages, etc. .

Le Président. - S'il n'y a pas d'autres questions, je remercie vivement $M$. WALDVOGEL et lui renouvelle nos félicitations pour l'aspect magistral de son exposé. Nous le remercions tous d'avoir bien voulu venir à Paris, bien que la Suisse ne soit pas loin de notre capitale. C'est un très grand plaisir et un honneur pour la Société Hydrotechnique de France de recevoir des collègues étrangers, surtout de votre valeur. 